\title{
Detection of the movement of the humerus during daily activity
}

\author{
Brian Coley $\cdot$ Brigitte M. Jolles · Alain Farron • \\ Kamiar Aminian
}

Received: 3 July 2008/Accepted: 10 February 2009/Published online: 10 March 2009

(C) International Federation for Medical and Biological Engineering 2009

\begin{abstract}
A new ambulatory technique for qualitative and quantitative movement analysis of the humerus is presented. $3 \mathrm{D}$ gyroscopes attached on the humerus were used to recognize the movement of the arm and to classify it as flexion, abduction and internal/external rotations. The method was first validated in a laboratory setting and then tested on 31 healthy volunteer subjects while carrying the ambulatory system during $8 \mathrm{~h}$ of their daily life. For each recording, the periods of sitting, standing and walking during daily activity were detected using an inertial sensor attached on the chest. During each period of daily activity the type of arm movement (flexion, abduction, internal/external rotation) its velocity and frequency (number of movement/hour) were estimated. The results showed that during the whole daily activity and for each activity (i.e. walking, sitting and walking) the frequency of internal/external rotation was significantly higher while the frequency of abduction was the lowest $(P<0.009)$. In spite of higher number of flexion, abduction and internal/external rotation in the dominant arm, we have not observed in our population a significant difference with the non-dominant arm, implying that in healthy subjects the arm dominance does not lie considerably on the number of movements. As expected, the frequency of the
\end{abstract}

B. Coley $\cdot$ K. Aminian $(\square)$

Laboratory of Movement Analysis and Measurement,

Ecole Polytechnique Fédérale de Lausanne (EPFL),

STI-Bat. ELH Station 11, 1015 Lausanne, Switzerland

e-mail: kamiar.aminian@epfl.ch

B. Coley

e-mail: bcoley@2-sight.com

B. M. Jolles - A. Farron

Département de l'Appareil Locomoteur,

Centre Hospitalier Universitaire Vaudois,

University of Lausanne, Lausanne, Switzerland movement increased from sitting to standing and from standing to walking, while we provide a quantitative value of this change during daily activity. This study provides preliminary evidence that this system is a useful tool for objectively assessing upper-limb activity during daily activity. The results obtained with the healthy population could be used as control data to evaluate arm movement of patients with shoulder diseases during daily activity.

Keywords Outcome evaluation .

Accelerometers and gyroscopes - Shoulder mobility . Ambulatory system

\section{Introduction}

Most quantitative approaches for shoulder movement analysis are performed in a laboratory setting where motion capture devices such as camera [9], electromagnetic [7], or electromyogram $[6,8]$ systems are used. Although very accurate and important for movement analysis their use is limited to the restricted volume of the laboratory. In order to quantify the movement that the subject can actually do during daily activity, it is useful to use an ambulatory device that can be carried by the subject during a whole day [1]. Long-term monitoring of shoulder movement (i.e. flexion, abduction and internal/external rotation) before and after intervention provides in this way objective outcomes for the evaluation of treatment. Such a movement monitoring can be performed using inertial sensors (e.g. accelerometers and gyroscope) attached on shoulder segments $[3,4]$. However, there is no study currently about the actual number of shoulder movements during daily activities, even though these studies can provide an objective evaluation of shoulder disease and its treatment. 
There is a general agreement that patients with rotator cuff impingement, adhesive capsulitis or glenohumeral degenerative diseases have a diminished arm flexion, abduction or internal/external rotation. In spite of complex studies, a precise evaluation of the shoulder movement based on the estimation of the number of movements per hour in real life conditions is still missing.

The goal of this study was twofold: first, validating an algorithm for the detection of the humerus movement of the shoulder (flexion, abduction and internal/external rotations), and second to evaluate the effectiveness of this algorithm during long-term measurements. By validating such an approach, we should provide a clinical tool that can be used to assess the shoulder's function and to find objective scores for outcome evaluation of a shoulder pathology treatment.

\section{Methods}

\subsection{Subjects and materials}

A total of 31 healthy subjects (32 years old $\pm 8 ; 18$ men, 13 women; 23 right handed, 8 left handed) were studied. Right or left handedness was established by a questionnaire. These data were used previously by Coley et al. $[3,4]$ in order to estimate the dominance of the arm and it position during daily activity. In order to show a clinical application of the proposed methods, one patient suffering from a rotator cuff disease implying a right shoulder supraspinatus rupture of $1 \mathrm{~cm}^{2}$ (48 years old, right-handed) was studied. In this study two inertial modules with three miniature capacitive gyroscopes (Analog device, ADXRS 250, $\pm 400 \mathrm{deg} / \mathrm{s}$ ) were fixed by a patch on each dorsal side of the distal humerus and one module with 3D gyroscopes and three miniature accelerometers (Analog device, ADXL 210, $\pm 5 \mathrm{~g}$ ) on the thorax [2] (Fig. 1). The sensors on the humerus were placed in such a manner to be aligned with the axis of humerus in order to measure the anterior flexion-extension (pitch), abduction-adduction (yaw) and internalexternal rotation (roll) of the shoulder, and the module fixed on the thorax was used for detecting daily activities (walking, sitting, standing) by using the method proposed by Najafi et al. [10, 11]. The signal from the sensors were amplified and low-pass filtered (cutoff frequency: $17 \mathrm{~Hz}$ ) to remove any electronic noise and artifacts. The sensors and their conditioning electronics were packaged in a very small box $(20 \times 20 \times 12 \mathrm{~mm})$. All signals were digitized at $200 \mathrm{~Hz}$ sampling rate and recorded by two synchronized data loggers (Physilog ${ }^{\circledR}$, BioAGM, $\mathrm{CH})$ carried on the subject's waist [2].

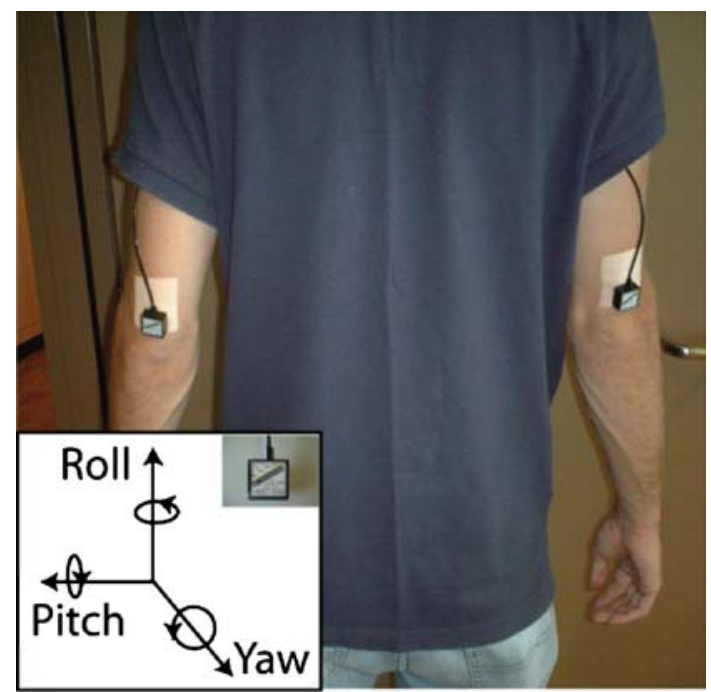

Fig. 1 Position of the inertial modules including 3D gyroscopes

\subsection{Body posture detection}

Body posture allocations (sitting and standing) as well as walking periods were detected by the trunk inertial module $[10,11]$. The time of sit-stand (respectively stand-sit) transition was detected from the patterns of angular tilt obtained from the gyroscope. Pattern recognition of the vertical acceleration allowed classifying the transition and deciding if the subject was in a standing or a sitting position. A walking period was defined as an interval with at least three gait cycles. The walking state was identified by analyzing the vertical accelerometer every five-seconds. The difference between the right and the left shoulder activity is shown for each period corresponding to sitting, standing and walking.

\subsection{Detection of the humerus movements}

3D angular velocities of the humerus were used to detect the movement and its axis of rotation. The pitch, roll and yaw angular velocities were associated respectively with flexion/extension, internal/external rotation and abduction/ adduction movement of the arm in agreement with ISB standardization proposal [13]. Figure 2 shows the three angular velocities recorded respectively for a flexion movement of $90^{\circ}$, an abduction of $90^{\circ}$ and an internal $/$ external rotation of $90^{\circ}$. During the flexion, the range of the pitch angular velocity was higher than the two other components (yaw and roll). Similar results can be observed for internal/external rotation (i.e. the range of the roll angular velocity was higher than yaw and pitch components) and for abduction (i.e. the range of the yaw angular velocity was higher than pitch and roll components). 


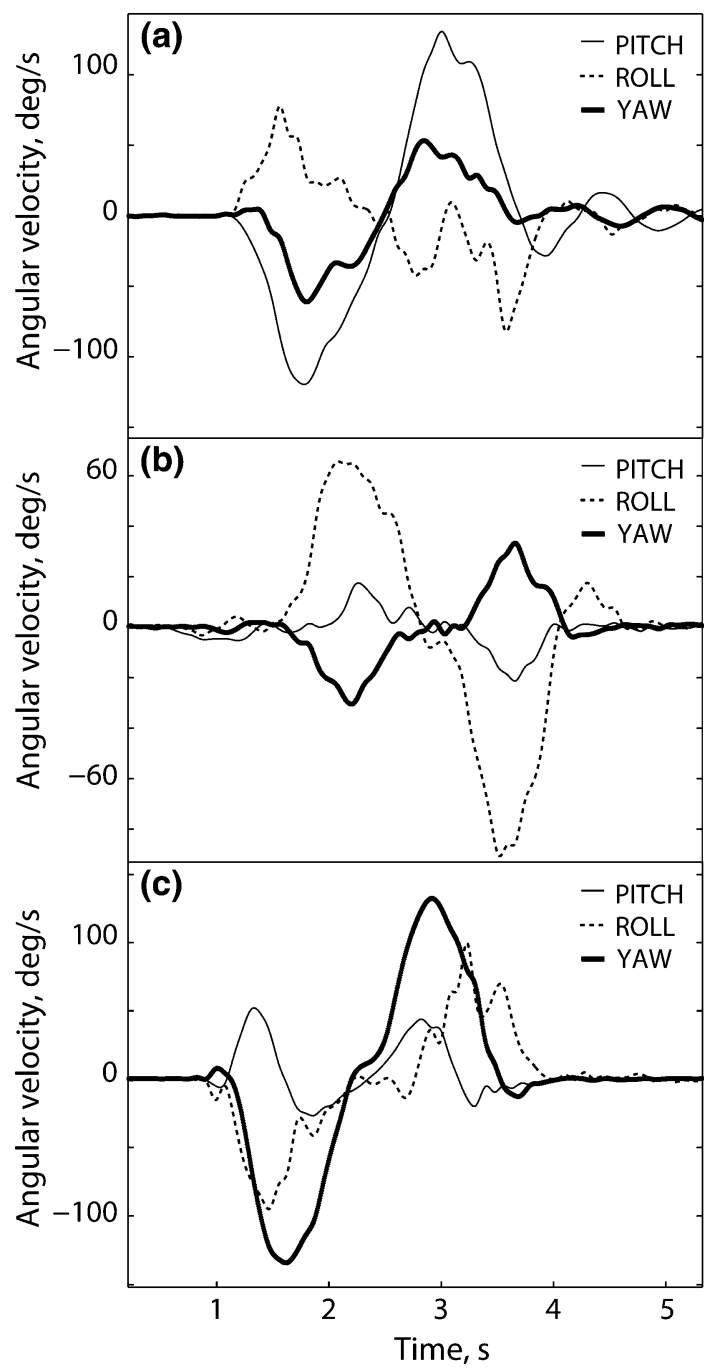

Fig. 2 Angular velocities (pitch, roll, yaw) detected by 3D gyroscope sensors for the flexion (a), the internal/external rotation (b) and the abduction (c)

To detect the shoulder movement, the absolute values of each component of angular velocity (pitch, roll, and yaw) was compared to a threshold (th). The shoulder was considered in movement if at least one component of angular velocity was higher than the the type of the movement (FE, AA, I.E) was estimated by considering the component of angular velocity with highest absolute angular velocity. If the highest angular velocity was pitch the movement was defined as a flexion-extension (FE). Similarly, if the highest absolute angular velocity belonged to yaw or roll, the movement was defined as abduction-adduction (AA) or internal/external rotation (I.E) respectively.

The threshold (th) was necessary to avoid the noise of the gyroscopes at rest and to decrease the false detections of the movement. The threshold (th) was adapted (adaptive threshold) every hour during the recording and was estimated for each subject and each humerus. To define th, we searched during each hour of recording all the positive peaks for each of the three angular velocities higher than $10 \%$ (almost still period of humerus). For each angular velocity, we calculated the average of the peaks. The threshold (th) was fixed to the minimum value of these averages.

\subsection{Validation setup}

To estimate the performance of the algorithm to correctly classify the type of the movement, the 31 subjects carried the system and were asked to perform at their desired velocity 2 flexions, 2 abductions and 2 internal/ external rotations with both arms while in the hospital. For the flexion and abduction subjects started with the arm along the body and performed a $90^{\circ}$ elevation while keeping the elbow unbent. The starting position of the internal/external rotation movement was with the upperarm along the body and the elbow flexed at $90^{\circ}$. Subjects were asked to perform an external rotation of $90^{\circ}$. A physiotherapist validated the movements done by the subject. In case of a wrong movement, the physiotherapist asked the subject to perform the movement again. The sensitivity (defined as the ability of the system to correctly identify the true movement) and the specificity (defined as the ability of the system to not generate false detection) were estimated. The sensitivity and the specificity were calculated as follows

Sensitivity was defined as

$\frac{\text { True positive }(\mathrm{TP})}{\text { True positive }(\mathrm{TP})+\text { False negative }(\mathrm{FN})} \times 100 \%$

Specificity was defined as

$\frac{\text { True negative }(\mathrm{TN})}{\text { True negative }(\mathrm{TN})+\text { False positive }(\mathrm{FP})} \times 100 \%$

For example, for the flexion movements the above parameters were defined as follow: the true positives were the numbers of true flexion detected by the algorithm. The false negatives were the numbers of undetected flexion. The true negatives were the numbers of other type of movement detected by the algorithm, which are not true flexion. The false positives were the numbers of false detection as flexion.

\subsection{Long-term measurement}

Each subject carried the ambulatory system during one day $(\sim 8 \mathrm{~h})$ at home or wherever he/she went. At the end of recording, the data was transferred to the computer for further analysis, and then the following parameters were estimated 
- The type of daily activity: sitting, standing and walking.

- The type of the movement: FE, AA and I.E.

- The frequency of each movement: the number of movements per hour recognized as flexion-extension $\left(N_{\mathrm{FE}}\right)$, abduction-adduction $\left(N_{\mathrm{AA}}\right)$ and internal/external rotation $\left(N_{\mathrm{IE}}\right)$. This frequency was estimated for all activity as well as for each type of daily activity.

- The frequency of each movement over three ranges of angular velocities: slow (less than $50 \mathrm{deg} / \mathrm{s}$ ), medium (between 50 and $100 \mathrm{deg} / \mathrm{s}$ ) and fast (higher than $100 \mathrm{deg} / \mathrm{s})$.

By definition frequency is normalized by the duration of each activity, it could therefore be a better metric that the number of movements that change with the duration of each activity.

The Wilcoxon ranked sum test was used as a nonparametric hypothesis test to show if there were any significant differences (at a significance level of 5\%) in the frequency of each movement.

\section{Results}

\subsection{Validation}

Table 1 summarizes the changes in sensitivity and specificity with the threshold obtained for different type of movement. Adaptive threshold provided excellent performances, while the minimum threshold (10 deg/s) corresponded to the worst cases. A fixed threshold of $33 \mathrm{deg} / \mathrm{s}$, corresponding to the average of all adaptive thresholds during long-term recording, was not satisfying either.

Table 1 Specificity and sensitivity for the detection of the flexion, abduction and internal/external rotation

\begin{tabular}{llrrrrrr}
\hline Threshold & Movement & TP & TN & FP & FN & $\begin{array}{l}\text { Sensitivity } \\
(\%)\end{array}$ & $\begin{array}{l}\text { Specificity } \\
(\%)\end{array}$ \\
\hline $10 \mathrm{deg} / \mathrm{s}$ & Flexion & 31 & 123 & 7 & 34 & 47 & 95 \\
& Abduction & 24 & 102 & 28 & 41 & 37 & 78 \\
& $\begin{array}{l}\text { Int/ext } \\
\text { rotation }\end{array}$ & 56 & 81 & 49 & 9 & 86 & 62 \\
$33 \mathrm{deg} / \mathrm{s}$ & Flexion & 41 & 122 & 8 & 24 & 63 & 94 \\
& Abduction & 36 & 108 & 22 & 29 & 55 & 83 \\
& Int/ext & 61 & 107 & 23 & 4 & 94 & 82 \\
& rotation & & & & & & \\
Adaptive & $\begin{array}{c}\text { Flexion } \\
\end{array}$ & 58 & 124 & 0 & 4 & 94 & 100 \\
& Abduction & 62 & 120 & 4 & 0 & 100 & 97 \\
& $\begin{array}{l}\text { Int/ext } \\
\text { rotation }\end{array}$ & 62 & 124 & 0 & 0 & 100 & 100 \\
& & & & & &
\end{tabular}

$T P$ True positive, $T N$ true negative, $F P$ false positive, $F N$ false negative

\subsection{Long-term measurement}

For each subject, walking, sitting and standing postures were recognized over a day $(\sim 8 \mathrm{~h})$ and for each posture the frequency of each movement was estimated for each humerus $\left(N_{\mathrm{FE}}, N_{\mathrm{AA}}, N_{\mathrm{IE}}\right)$. Table 2 summarizes the value of the frequency over all activity by dividing the number of movement by the duration of measurement $(\sim 8 \mathrm{~h})$. Table 3 reports the value of frequency for each posture, by

Table 2 Frequency (number per hour) of flexion $\left(N_{\mathrm{FE}}\right)$, abduction $\left(N_{\mathrm{AA}}\right)$ and internal/external rotation $\left(N_{\mathrm{IE}}\right)$ for all activities for the right handed subjects (r1 to r23) and left handed subjects (11-18) with their mean and standard deviation (SD)

\begin{tabular}{|c|c|c|c|c|c|c|}
\hline \multirow[t]{2}{*}{ Subject } & \multicolumn{2}{|l|}{$N_{\mathrm{FE}}$} & \multicolumn{2}{|l|}{$N_{\mathrm{AA}}$} & \multicolumn{2}{|l|}{$N_{\mathrm{IE}}$} \\
\hline & Right & Left & Right & Left & Right & Left \\
\hline r1 & 157 & 121 & 73 & 47 & 272 & 259 \\
\hline r2 & 150 & 181 & 76 & 59 & 291 & 273 \\
\hline r3 & 129 & 105 & 44 & 35 & 209 & 168 \\
\hline $\mathrm{r} 4$ & 124 & 109 & 59 & 70 & 301 & 244 \\
\hline r5 & 87 & 95 & 54 & 40 & 251 & 188 \\
\hline r6 & 123 & 72 & 37 & 33 & 213 & 113 \\
\hline r7 & 136 & 189 & 90 & 56 & 298 & 274 \\
\hline r8 & 99 & 96 & 38 & 38 & 237 & 219 \\
\hline r9 & 131 & 119 & 36 & 35 & 185 & 165 \\
\hline r10 & 86 & 68 & 39 & 33 & 160 & 103 \\
\hline r11 & 165 & 154 & 68 & 57 & 309 & 213 \\
\hline $\mathrm{r} 12$ & 215 & 199 & 96 & 109 & 476 & 444 \\
\hline r13 & 161 & 122 & 69 & 87 & 308 & 306 \\
\hline r14 & 246 & 222 & 140 & 139 & 521 & 473 \\
\hline r15 & 210 & 175 & 81 & 102 & 406 & 388 \\
\hline r16 & 153 & 173 & 77 & 65 & 406 & 310 \\
\hline r17 & 134 & 139 & 66 & 53 & 311 & 283 \\
\hline r18 & 205 & 146 & 80 & 67 & 420 & 396 \\
\hline r19 & 252 & 287 & 152 & 103 & 492 & 476 \\
\hline r20 & 183 & 150 & 93 & 70 & 333 & 322 \\
\hline r21 & 210 & 229 & 112 & 114 & 534 & 503 \\
\hline r22 & 196 & 176 & 104 & 80 & 438 & 377 \\
\hline r23 & 234 & 224 & 87 & 97 & 539 & 491 \\
\hline Mean & 165 & 154 & 77 & 69 & 344 & 304 \\
\hline SD & 49 & 55 & 31 & 30 & 116 & 122 \\
\hline 11 & 156 & 209 & 86 & 112 & 325 & 342 \\
\hline 12 & 129 & 129 & 70 & 65 & 306 & 326 \\
\hline 13 & 206 & 163 & 83 & 90 & 396 & 407 \\
\hline 14 & 141 & 146 & 68 & 95 & 270 & 270 \\
\hline 15 & 200 & 169 & 89 & 94 & 388 & 408 \\
\hline 16 & 79 & 93 & 47 & 50 & 190 & 198 \\
\hline 17 & 245 & 265 & 98 & 141 & 360 & 376 \\
\hline 18 & 201 & 244 & 99 & 107 & 388 & 406 \\
\hline Mean & 170 & 177 & 80 & 94 & 328 & 342 \\
\hline $\mathrm{SD}$ & 53 & 58 & 17 & 28 & 71 & 75 \\
\hline
\end{tabular}


Table 3 Frequency of the movement $N_{\mathrm{FE}}, N_{\mathrm{AA}}$ and $N_{\mathrm{IE}}$ for the right and left handed subjects

\begin{tabular}{|c|c|c|c|c|c|c|c|c|c|c|c|c|c|c|c|c|c|c|}
\hline \multirow[t]{2}{*}{ Subject } & \multicolumn{6}{|l|}{ Walk } & \multicolumn{6}{|l|}{ Sit } & \multicolumn{6}{|l|}{ Stand } \\
\hline & $\begin{array}{l}N_{\mathrm{FE}} \\
\text { right }\end{array}$ & Left & $\begin{array}{l}N_{\mathrm{AA}} \\
\text { right }\end{array}$ & Left & $N_{\text {IE right }}$ & Left & $\begin{array}{l}N_{\mathrm{FE}} \\
\text { right }\end{array}$ & Left & $\begin{array}{l}N_{\mathrm{AA}} \\
\text { right }\end{array}$ & Left & $\begin{array}{l}N_{\mathrm{IE}} \\
\text { right }\end{array}$ & Left & $\begin{array}{l}N_{\mathrm{FE}} \\
\text { right }\end{array}$ & Left & $\begin{array}{l}N_{\mathrm{AA}} \\
\text { right }\end{array}$ & Left & $\begin{array}{l}N_{\mathrm{IE}} \\
\text { right }\end{array}$ & Left \\
\hline r1 & 464 & 280 & 250 & 175 & 795 & 822 & 113 & 89 & 52 & 25 & 210 & 178 & 142 & 122 & 60 & 45 & 231 & 234 \\
\hline r2 & 349 & 493 & 206 & 145 & 708 & 693 & 72 & 92 & 29 & 27 & 133 & 119 & 132 & 133 & 66 & 52 & 255 & 236 \\
\hline r3 & 378 & 568 & 209 & 127 & 745 & 779 & 97 & 47 & 19 & 22 & 129 & 83 & 143 & 119 & 57 & 41 & 253 & 204 \\
\hline r4 & 380 & 373 & 184 & 288 & 747 & 740 & 67 & 52 & 35 & 30 & 199 & 119 & 103 & 89 & 42 & 47 & 246 & 226 \\
\hline r5 & 332 & 450 & 283 & 180 & 799 & 807 & 34 & 27 & 13 & 16 & 129 & 60 & 70 & 65 & 33 & 23 & 205 & 148 \\
\hline r6 & 723 & 668 & 231 & 296 & 919 & 863 & 82 & 31 & 27 & 16 & 156 & 55 & 114 & 75 & 25 & 30 & 219 & 130 \\
\hline r7 & 349 & 581 & 256 & 140 & 765 & 816 & 83 & 98 & 43 & 27 & 152 & 120 & 104 & 139 & 78 & 52 & 267 & 239 \\
\hline r8 & 396 & 244 & 175 & 137 & 697 & 758 & 75 & 80 & 27 & 33 & 213 & 187 & 85 & 89 & 31 & 28 & 182 & 170 \\
\hline r9 & 712 & 688 & 261 & 204 & 856 & 872 & 47 & 46 & 11 & 14 & 91 & 67 & 115 & 96 & 24 & 27 & 163 & 140 \\
\hline r10 & 496 & 541 & 341 & 343 & 805 & 812 & 70 & 34 & 25 & 11 & 111 & 42 & 41 & 30 & 10 & 9 & 101 & 52 \\
\hline r11 & 590 & 607 & 251 & 260 & 858 & 759 & 94 & 80 & 43 & 34 & 206 & 112 & 160 & 149 & 56 & 39 & 303 & 224 \\
\hline r12 & 440 & 426 & 204 & 288 & 837 & 841 & 88 & 100 & 31 & 35 & 259 & 203 & 187 & 153 & 88 & 83 & 403 & 388 \\
\hline r13 & 498 & 396 & 201 & 318 & 871 & 898 & 95 & 66 & 44 & 39 & 185 & 170 & 163 & 127 & 66 & 96 & 321 & 332 \\
\hline r14 & 424 & 440 & 265 & 269 & 783 & 808 & 136 & 101 & 60 & 69 & 301 & 232 & 165 & 140 & 93 & 88 & 378 & 331 \\
\hline r15 & 484 & 389 & 207 & 233 & 778 & 751 & 99 & 87 & 31 & 37 & 224 & 193 & 131 & 111 & 49 & 73 & 286 & 284 \\
\hline r16 & 531 & 607 & 252 & 184 & 830 & 883 & 87 & 108 & 40 & 34 & 301 & 201 & 140 & 144 & 76 & 68 & 386 & 285 \\
\hline r17 & 530 & 564 & 254 & 184 & 822 & 863 & 46 & 39 & 21 & 18 & 142 & 109 & 96 & 103 & 51 & 42 & 275 & 243 \\
\hline r18 & 361 & 261 & 125 & 104 & 716 & 680 & 90 & 63 & 34 & 29 & 203 & 184 & 131 & 91 & 57 & 48 & 269 & 254 \\
\hline r19 & 544 & 597 & 272 & 216 & 878 & 889 & 102 & 117 & 80 & 46 & 251 & 209 & 188 & 218 & 120 & 77 & 389 & 378 \\
\hline r20 & 604 & 559 & 265 & 219 & 878 & 841 & 79 & 58 & 38 & 32 & 179 & 165 & 132 & 99 & 82 & 52 & 261 & 265 \\
\hline r21 & 440 & 471 & 239 & 211 & 862 & 886 & 119 & 133 & 61 & 58 & 356 & 283 & 156 & 171 & 84 & 93 & 432 & 412 \\
\hline r22 & 412 & 434 & 242 & 184 & 843 & 793 & 124 & 96 & 60 & 49 & 266 & 199 & 182 & 166 & 99 & 73 & 440 & 398 \\
\hline r23 & 416 & 466 & 160 & 199 & 877 & 900 & 109 & 90 & 31 & 25 & 262 & 196 & 173 & 155 & 66 & 71 & 415 & 366 \\
\hline Mean & 472 & 483 & 232 & 213 & 812 & 815 & 87 & 75 & 37 & 32 & 202 & 152 & 133 & 121 & 61 & 55 & 290 & 258 \\
\hline SD & 109 & 124 & 46 & 65 & 61 & 64 & 25 & 30 & 17 & 14 & 68 & 64 & 38 & 41 & 27 & 24 & 92 & 95 \\
\hline 11 & 560 & 591 & 290 & 281 & 827 & 825 & 118 & 188 & 53 & 102 & 276 & 315 & 165 & 232 & 98 & 130 & 382 & 399 \\
\hline 12 & 393 & 387 & 178 & 189 & 785 & 775 & 81 & 84 & 47 & 41 & 200 & 220 & 113 & 108 & 66 & 59 & 294 & 319 \\
\hline 13 & 512 & 439 & 237 & 262 & 853 & 789 & 52 & 38 & 21 & 19 & 117 & 123 & 164 & 123 & 57 & 64 & 339 & 369 \\
\hline 14 & 584 & 657 & 325 & 332 & 866 & 876 & 93 & 101 & 37 & 82 & 194 & 212 & 128 & 118 & 64 & 76 & 274 & 250 \\
\hline 15 & 546 & 403 & 193 & 229 & 860 & 886 & 95 & 98 & 48 & 41 & 194 & 217 & 137 & 120 & 69 & 73 & 304 & 317 \\
\hline 16 & 236 & 379 & 210 & 198 & 687 & 792 & 43 & 44 & 21 & 25 & 101 & 96 & 126 & 120 & 56 & 67 & 260 & 260 \\
\hline 17 & 543 & 544 & 198 & 195 & 721 & 693 & 89 & 174 & 47 & 77 & 184 & 241 & 218 & 212 & 87 & 149 & 319 & 327 \\
\hline 18 & 563 & 589 & 309 & 205 & 859 & 822 & 81 & 93 & 37 & 47 & 168 & 216 & 162 & 225 & 74 & 107 & 360 & 362 \\
\hline Mean & 492 & 499 & 242 & 236 & 807 & 807 & 82 & 103 & 39 & 54 & 179 & 205 & 152 & 157 & 72 & 90 & 316 & 325 \\
\hline SD & 119 & 109 & 57 & 51 & 70 & 61 & 24 & 54 & 12 & 30 & 54 & 68 & 33 & 55 & 15 & 34 & 42 & 52 \\
\hline
\end{tabular}

dividing the number of movement in each posture by the total time of this posture during measurement. The average of the frequency was higher for the right humerus (flexion right: 165; flexion left: 154; abduction right: 77; abduction left: 69; internal/external rotation right: 344; internal/ external rotation left: 304$)$ for the right handed subjects $(n=23)$. While for the left handed subjects, the average of the frequency was higher for the left humerus $(n=8)$ (flexion right: 170; flexion left: 177; abduction right: 80; abduction left: 94; internal/external rotation right: 328; internal/external rotation left: 342) (Table 3). However, statistical tests showed that the dominant shoulder and the non-dominant shoulder had no significant difference $(P>0.1)$ for the frequency of flexions $\left(N_{\mathrm{FE}}\right)$, abductions $\left(N_{\mathrm{AA}}\right)$ and internal/external rotations $\left(N_{\mathrm{IE}}\right)$ in the sitting and standing posture (Table 2). Moreover, there was no significant difference $(P>0.3)$ between the dominant and non-dominant shoulder in the gait.

The frequency of the movement was significantly higher for walking compared to sitting and standing and significantly lower in sitting compared to standing $(P<0.008)$. For all postures, as well as during the whole daily activity, 
Fig. 3 Distribution of the movements (frequency vs. range of angular velocities values) for the control group and for a right-handed patient suffering from rotator cuff disease in the right shoulder. a All activities b walking, c standing, $\mathbf{d}$ sitting. Slow (up to $50 \mathrm{deg} / \mathrm{s}$ ), medium (between 50 and $100 \mathrm{deg} / \mathrm{s}$ ) and fast (more than $100 \mathrm{deg} / \mathrm{s}$ )

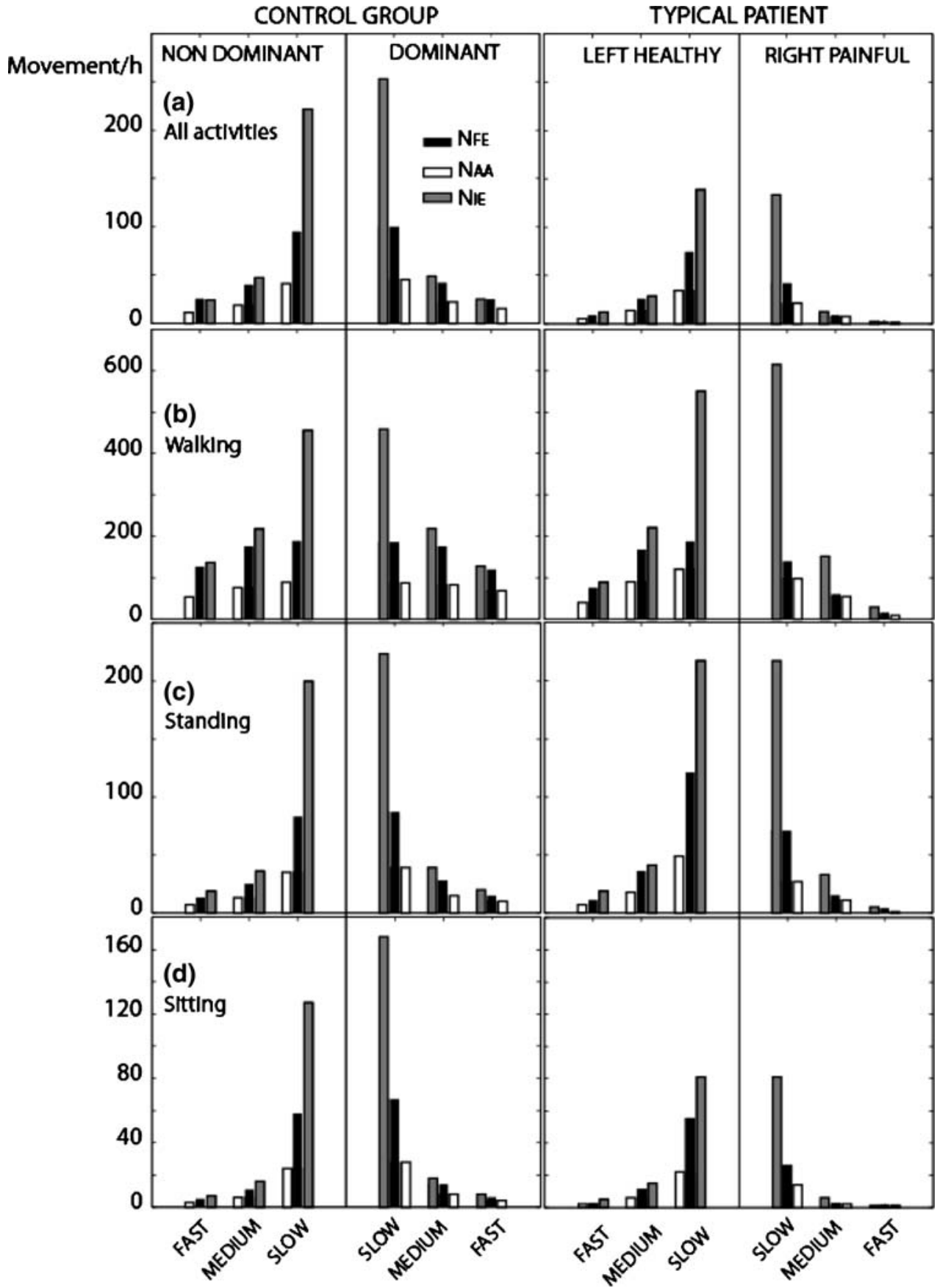

we found a significantly higher frequency of movement in internal/external rotation and the lowest frequency of movement in abduction $(P<0.009)$.

\subsection{The change of humerus velocity due to pain}

Another aspect, which could be studied in shoulder pathology is the change of humerus velocity due to pain. To highlight this point we have plotted in Fig. 3 for all control subjects the distribution of each movement per hour in three ranges of angular velocity: slow (up to $50 \mathrm{deg} / \mathrm{s}$ ), medium (between $50 \mathrm{deg} / \mathrm{s}$ and $100 \mathrm{deg} / \mathrm{s}$ ) and fast (more than $100 \mathrm{deg} / \mathrm{s}$ ). For comparison we have performed a long-term recording with right-handed patient suffering from a rotator cuff tear in the right shoulder.

\section{Discussion}

In this study, an ambulatory system was proposed to evaluate the mobility of the shoulder during daily physical activity. The method used the speed of rotation of the 
humerus and not the orientation of the humerus, avoiding in this way any noise and drift due to time integration of the gyroscope signals to find angles [5]. The performance of the method to detect the movement and classify the movement as FE, AA and IE lies on the adequate choice of the threshold (th). By using an adaptive threshold we provided a better performance since th was modified based on the amplitude of angular velocity in each window of $1 \mathrm{~h}$. We also evaluated the change in th during the validation setup and for the long-term measurement of each subject. We noticed that the mean th (over $8 \mathrm{~h}$ and all subjects) was different for the validation phase (th $=52 \pm 7 \mathrm{deg} / \mathrm{s}$ ) where the movement was imposed and the long-term measurement (th $=33 \pm 3 \mathrm{deg} / \mathrm{s}$ ) where the movement was spontaneous. To show the efficacy of the adaptive threshold, we calculated the specificity and the sensitivity in the validation phase with a fixed threshold of $33 \mathrm{deg} / \mathrm{s}$ obtained from the long-term measurement. The sensitivities and specificities obtained were lower than those with the adaptive threshold.

Based on 3D gyroscopes on both humeri, our method has not only qualified the type of the movement but also quantified the frequency and the speed of the movement in FE, AA and I.E and their change between dominant and non-dominant shoulder during daily activity. Although in our healthy population the dominant shoulder has a higher frequency of the movement compared to non-dominant side, we have not observed a significant level of difference $(P>0.1)$. These results would also imply that in healthy subjects the arm predominance does not lie considerably on the number of movements in the arm. If we compare this finding with our recent results, where the dominant and non-dominant arms have significantly different intensity during movement [3], we can conclude that the frequency should be estimated in terms of the velocity of the movement as proposed in Sect. 2.5 and shown in Fig. 3.

We observed that the frequency of the movement increased from sitting to standing and from standing to walking. Although this would be expected, since we have more activities during standing and walking compared to sitting, the present study provided a quantitative value of these changes. In addition, in daily activities the most common movement was the internal/external rotation and the less frequent one was the abduction. While the movement of flexion was important during the gait for example, the movements of internal/external rotation were performed during all daily tasks like working in an office, cleaning a table etc. Our proposed technique could be useful to determine daily physical activities which require the most flexion, abduction or internal/external rotations.

As far as our clinical case is concerned, we can conclude that our right-handed patient with a painful right shoulder performed more movements with the left shoulder (nondominant) than the right shoulder (dominant) during his daily activities (Fig. 3). The frequency of the movement distribution in the healthy non-dominant shoulder is close to the non-dominant shoulder of the control population. The frequency of the movement and the velocity distribution for the patient is higher for the left healthy shoulder than the right painful shoulder. We can expect that this tendency should be logically reversed after the surgery of the shoulder and recovery. Moreover, we can observe more difference in medium and fast movement than slow movement. This again assumes an increase of the frequency of the faster movement after surgery. Futures studies with higher number of patients are needed to confirm these hypotheses and show the shoulder function evolution after surgery.

A potential extrinsic confounding parameter could be the external charge (such as a bag or a suitcase) that the subject can carry during his daily activity with his arm. For example, it is not possible with the proposed method to determine whether a subject is performing ordinary walking or carrying a bag while walking. We can expect that by carrying a bag, the number of flexion will decrease and appears like a disease. Calibrating the ordinary walking of a subject at the beginning of a measurement period or using electromyogram recordings might be a solution. A method which is able to give $3 \mathrm{D}$ angles during the daily activity or the intensity of the movement will be complementary to this study. Indeed, the addition of the angles value or the power of the movement with the type of the rotation could illustrate more difference between the left and right shoulder.

\section{Conclusion}

Based on the shoulder kinematics, we were able to find the numbers of flexions, abductions and internal/external rotations of the humerus during daily activity for a healthy population. Our proposed system appears especially promising for long-term monitoring: the sensors have low power consumption $(17 \mathrm{~mA})$ and with standard batteries the system allows one to record up to $8 \mathrm{~h}$ on a memory of 512 MBytes. Monitoring the subjects in their usual environment with minimal interference is therefore possible in contrast with other systems that require a laboratory setting. These results were very encouraging for future evaluation of patients with shoulder injuries. This study will be also very helpful to simulate the performance of the new design of shoulder prosthesis and implants (in laboratory or numerically) because it can provide the actual shoulder movement during daily activity [12]. 
Acknowledgments The authors are grateful to J. Gramiger and P. Morel for the design of the ambulatory system and to C. Pichonnaz, J.P. Bassin and A. Poloni, for their help during the measurement. This work was supported by Swiss National Foundation (PNR 53) Grant no $405340-104752 / 1$.

\section{References}

1. Aminian K (2006) Human movement capture and their clinical applications, In: Begg RK, Palaniswami M (eds) Computational intelligence for movement sciences: neural networks, support vector machines and other emerging techniques. Idea Group Inc., USA, pp 101-138

2. Coley B, Jolles BM, Farron A, Bourgeois A, Nussbaumer F, Pichonnaz C, Aminian K (2007) Outcome evaluation in shoulder surgery using 3D kinematics sensors. Gait Posture 25:523-532

3. Coley B, Jolles BM, Farron A, Pichonnaz C, Bassin JP, Aminian K (2008) Estimating the upper-limb dominant segment during daily activity. Gait Posture 27:368-375

4. Coley B, Jolles BM, Farron A, Aminian K (2008) Arm position during daily activity. Gait Posture 28:581-587

5. Favre J, Jolles B, Siegrist O, Aminian A (2006) Quaternion-based fusion of gyroscopes and accelerometers to improve $3 \mathrm{D}$ angle measurement. Electron Lett 42:11

6. Illyes A, Kiss RM (2006) Electromyographic analysis in patients with multidirectional shoulder instability during pull, forward punch, elevation and overhead throw. Knee Surg Sports Traumatol Arthrosc 10:163-167
7. Meskers CG, Vermeulen HM, de Groot JH, r Helm FC, Rozing PM (1998) 3D shoulder position measurements using a sixdegree-of-freedom electromagnetic tracking device. Clin Biomech 13:280-292

8. Meskers CG, de Groot JH, Arwert HJ, Rozendaal LA, Rozing PM (2004) Reliability of force direction dependent EMG parameters of shoulder muscles for clinical measurements. Clin Biomech. 9:13-20

9. Murray IA, Johnson GR (2004) A study of the external forces and moments at the shoulder and elbow while performing every day tasks. Clin Biomech 19:586-594

10. Najafi B, Aminian K, Loew F, Blanc Y, Robert PA (2002) Measurement of stand-sit and sit-stand transitions using a miniature gyroscope and its application in fall risk evaluation in the elderly. IEEE Trans Biomed Eng 49:843-851

11. Najafi B, Aminian K, Paraschiv-Ionescu A, Loew F, Bula CJ, Robert P (2003) Ambulatory system for human motion analysis using a kinematic sensor: monitoring of daily physical activity in the elderly. Biomed Eng IEEE Trans 50:11-723

12. Terrier A, Reist A, Vogel A, Farron A (2007) Effect of supraspinatus deficiency on humerus translation and glenohumeral contact force during abduction. Clin Biomech 22(6):645-651

13. Wu G, van der Helm FC, Veeger HE, Makhsous M, Van Roy P, Anglin C, Nagels J, Karduna AR, McQuade K, Wang X, Werner FW, Buchholz B (2005) ISB recommendation on definitions of joint coordinate systems of various joints for the reporting of human joint motion-Part II: shoulder, elbow, wrist and hand. J Biomech 38:981-992 